\title{
Integrazione geometrica di alcune equazioni differenziali.
}

\author{
(Di Geminiano Pirondini, a Parma.)
}

Se l'integrazione di una certa equazione differenziale, o risolto oon un secondo metodo è ridotto a sole quadrature, pud avvenire che, col confronto delle due soluzioni, si riesca ad ottenere l'integrale generale di quell'equazione differenziale.

Per rendere ciò manifesto con un esempio, proponiamoci il seguente problema: Determinare una linea a doppia curvatura colle due condizioni:

$1 .^{\circ}$ che il raggio vettore $R$, che dall'origine degli assi va a un punto arbitrario della linea, sia una funzione nota dell'arco;

$2 .^{\circ}$ che il coseno dell'angolo $\omega$, sotto il quale la linea sega $i$ meridiani della superficie generata dalla medesima, nella sua rotazione attorno all'asse delle z, sia una funzione nota dell'arco.

Le coordinate dei punti di una linea qualsivoglia si possono esprimere, in funzione dell'arco, per mezzo delle equazioni:

$$
\begin{aligned}
& x=\rho \cdot \cos \left(\int \frac{\sqrt{1-\rho^{\prime 2}-\lambda^{\prime 2}}}{\rho} \cdot d s\right) \\
& y=\rho \cdot \operatorname{sen}\left(\int \frac{\sqrt{1-\rho^{\prime 2}-\lambda^{\prime 2}}}{\rho} \cdot d s\right) \\
& z=\lambda(s) .
\end{aligned}
$$

Ed allora, essendo:

si deduce :

$$
\cos \omega=\sqrt{\rho^{2}+\lambda^{\prime 4}}
$$

$$
\lambda=\int \sqrt{\cos ^{2} \omega-\rho^{\prime 2}} \cdot d s
$$

potendosi evidentemente mettere a zero la costante arbitraria (additiva) che viene dall'integrazione. 
Notando che

$$
\rho^{2}+z^{2}=R^{2}
$$

se si tiene conto dell'espressione (2) di $\lambda$ e si applicano le equazioni (1), si trova che la linea richiesta è rappresentata dalle equazioni:

$$
\begin{gathered}
x=\rho \cdot \cos \left(\int \frac{\operatorname{sen} \omega}{\rho} d s\right), \quad y=\rho \cdot \operatorname{sen}\left(\int \frac{\operatorname{sen} \omega}{\rho} d s\right), \\
z=\int \sqrt{\cos ^{2} \omega-\rho^{\prime 2}} d s,
\end{gathered}
$$

dove $\rho$ è definita in funzione di s per mezzo dell'equazione differenziale:

$$
\rho^{\prime 2}-\frac{2 R^{\prime}}{R} \cdot \rho \rho^{\prime}+\frac{\cos ^{2} \omega}{R^{2}} \rho^{2}=\cos ^{2} \omega-R^{\prime 2} .
$$

Le coordinate dei punti di una linea possono esprimersi, in funzione dell'arco, anche per mezzo delle equazioni:

$$
\left\{\begin{array}{l}
x=R \operatorname{sen} \varphi \cdot \cos \left(\int \frac{\sqrt{1-R^{\prime 2}-R^{2} \varphi^{\prime 2}}}{R \operatorname{sen} \varphi} \cdot d s\right) \\
y=R \operatorname{sen} \varphi \cdot \operatorname{sen}\left(\int \frac{\sqrt{1-R^{\prime 2}-R^{2} \varphi^{\prime 2}}}{R \operatorname{sen} \varphi} \cdot d s\right) \\
z=R \cos \varphi .
\end{array}\right.
$$

In questa ipotesi si trova:

$$
\cos \omega=\sqrt{R^{\prime 2}+R^{2} \varphi^{\prime 2}},
$$

da cui si ricava:

$$
\varphi=\int \frac{\sqrt{\cos ^{2} \omega-R^{\prime 2}}}{R} \cdot d s+c,
$$

con $c$ costante arbitraria.

Si vede quindi che la linea domandata è anche definita dalle equazioni:

$$
\left\{\begin{array}{l}
x=R \cdot \operatorname{sen}\left(\int \frac{\sqrt{\cos ^{2} \omega-R^{\prime 2}}}{R} d s+c\right) \cdot \cos \left\{\int \frac{\operatorname{sen} \omega \cdot d s}{R \cdot \operatorname{sen}\left(\int \frac{\sqrt{\cos ^{2} \omega-R^{\prime 2}}}{R} \cdot d s+c\right)}\right\} \\
y=R \cdot \operatorname{sen}\left(\int \frac{\sqrt{\cos ^{2} \omega-R^{\prime 2}}}{R} d s+c\right) \cdot \operatorname{sen}\left\{\int \frac{\operatorname{sen} \omega \cdot d s}{R \cdot \operatorname{sen}\left(\int \frac{\sqrt{\cos ^{2} \omega-R^{\prime 2}}}{R} \cdot d s+c\right)}\right\} \\
z=R \cdot \cos \left(\int \frac{\sqrt{\cos ^{2} \omega-R^{\prime 3}}}{R} d s+c\right) .
\end{array}\right.
$$


Basta confrontare queste equazioni colle (3) per riconoscere che l'integrale generale dell'equazione (4) è:

$$
\rho=R \cdot \operatorname{sen}\left(\int \frac{\sqrt{\cos ^{2} \omega-R^{\prime 2}}}{R} d s+c\right) .
$$

Si verifica poi, colla prova diretta, che tale proprietà non cessa di sussistere se nella (4) l'arco $s$ si sostituisce con una variabile indipendente qualunque, spogliando anche le funzioni arbitrarie $\omega, R$ di quel particolare significato geometrico che era loro stato attribuito. Cambiando allora $s$ e $p$ rispettivamente in $x$ ed $y$, e ponendo inoltre:

$$
M=-\frac{2 R^{\prime}}{R}, \quad N=\frac{\cos ^{2} \omega}{R^{2}}, \quad P=\cos ^{2} \omega-R^{\prime 2},
$$

la (4) può scriversi :

$$
\left(\frac{d y}{d x}\right)^{2}+M \cdot y \frac{d y}{d x}+N y^{2}=P
$$

E siccome dalle (5) si ricava:

$$
R=e^{-\frac{1}{2} \int M d x}, \quad \cos \omega=\sqrt{N} \cdot e^{-\frac{1}{2} \int M d d x}, \quad P=\left(N-\frac{M^{2}}{4}\right) e^{-\int \Delta I d x},
$$

si ha il teorema:

L'integrale generale dell'equarione differenziale del primo ordine e del secondo grado:

$$
\left(\frac{d y}{d x}\right)^{2}+M \cdot y \frac{d y}{d x}+N \cdot y^{2}=\left(N-\frac{M^{2}}{4}\right) e^{-\int M d x}
$$

(dove $M$ ed $N$ sono due funzioni arbitrarie della sola $x$ ) è il seguente:

$$
y=e^{-\frac{1}{2} \int M d x} \cdot \operatorname{sen}\left(\int \sqrt{N-\frac{M^{2}}{4}} d x+c\right)
$$

Parma, settembre 1903. 